\title{
Oral Hygiene Status, Periodontal Status, and Periodontal Treatment Needs among Institutionalized Intellectually Disabled Subjects in Kolhapur District, Maharashtra, India
}

\author{
Nilima S. Kadam, ${ }^{1}$ Rahul Patil, ${ }^{1}$ Abhijit N. Gurav, ${ }^{1}$ Yojana Patil, ${ }^{2}$ Abhijeet Shete, ${ }^{1}$ \\ Ritam Naik Tari, ${ }^{1}$ Dhanashree Agarwal, ${ }^{1}$ D. T. Shirke, ${ }^{3}$ and Prashant Jadhav ${ }^{4}$ \\ ${ }^{1}$ Department of Periodontology, Tatyasaheb Kore Dental College \& Research Centre, New Pargaon, Kolhapur, \\ Maharashtra 416137, India \\ ${ }^{2}$ Department of Public Health Dentistry, Tatyasaheb Kore Dental College \& Research Centre, Kolhapur, Maharashtra, India \\ ${ }^{3}$ Department of Statistics, Shivaji University, Kolhapur, Maharashtra, India \\ ${ }^{4}$ Department of Prosthodontics, Tatyasaheb Kore Dental College \& Research Centre, Kolhapur, Maharashtra, India
}

Correspondence should be addressed to Nilima S. Kadam; dr.nilima15@gmail.com

Received 30 August 2014; Revised 10 November 2014; Accepted 11 November 2014; Published 7 December 2014

Academic Editor: Tetsuo Kobayashi

\begin{abstract}
Copyright (C) 2014 Nilima S. Kadam et al. This is an open access article distributed under the Creative Commons Attribution License, which permits unrestricted use, distribution, and reproduction in any medium, provided the original work is properly cited.
\end{abstract}

\begin{abstract}
The aim of the study was to determine influence of intellectually disability (ID) on oral hygiene status (OHS), periodontal status (PS), and periodontal treatment needs (PTNs) and investigate the association of these clinical parameters with various sociodemographic and clinical variables among multicentric institutionalized ID subjects aged 5-55 years in Kolhapur district, Maharashtra, India. This descriptive cross-sectional study was conducted by both questionnaire and clinical examination. OHS, PS, and PTNs of these subjects were assessed by recording Simplified Oral Hygiene Index (OHI-S) and Community Periodontal Index of Treatment Needs, respectively. Analysis of variance and multiple logistic and stepwise linear regression analysis were used for statistical analysis. Of the 576 subjects examined, the mean age of the subjects was $17.50 \pm 7.4$ years. Subjects with profound level of ID had the highest mean OHI-S, PS, and PTNs scores of $4.47 \pm 1.04,2.84 \pm 0.78$, and $2.25 \pm 0.44$, respectively, as compared with other ID groups $(P<0.0001)$. The present study signified that ID subjects had poor OHS and PS with the highest PTNs and were influenced by age $(P<0.0001)$, degree of ID $(P<0.0001)$, SES, location, institution type, and parent's level education. The present study underlines a considerable need for prevention and treatment of periodontal disease among ID subjects in India.
\end{abstract}

\section{Introduction}

Oral health has been defined as "the standard of health of the oral and related tissues which enables an individual to eat, speak and socialize without active disease, discomfort and embarrassment and which contributes to general well being" [1]. The oral health needs of individuals with intellectual disability (ID) are multifaceted, persistent, and expensive, and for various reasons they are not satisfactorily met. Information concerning the oral hygiene status, periodontal status, and periodontal treatment needs of subjects with ID is essential to develop compensatory strategies to promote and protect the oral health of this vulnerable population and create best practices for inclusion in dental treatment guidelines.

ID refers to "a group of developmental conditions characterized by significant impairment of cognitive functions, which are associated with limitations of learning, adaptive behaviour and skills." The disorder is considered chronic and originates before the age of 18 [2]. ID individuals have an intelligent quotient (IQ) score of about 70 or below. ID often cooccurs with other mental conditions such as Down's syndrome (DS), cerebral palsy (CP), autism spectrum disorders, epilepsy, traumatic brain injury, Alzheimer's disease, attention deficit hyperactivity disorder (ADHD), developmental coordination disorder, learning disabilities, 
and sensory impairments. ID is divided based on IQ score measured through standardized general aptitude evaluation tools, such as the Wechsler Intelligence Scales or StanfordBinet Intelligence Scales. Various degrees of ID are mild (50 to 69 ), moderate (35 to 49 ), severe (20 to 34 ), and profound (less than 20) categories. IQ has been used as the sole criterion for deciding educational programs for these subjects $[2,3]$.

The prevalence of ID is $10.37 / 1000$ population. The prevalence is higher among children and adolescents in low and middle income group countries [4]. In India, according to the National Sample Survey Organization (NSSO), the number of disabled persons is approximated to be about $1.8 \%$ of the total population, while the ID population is estimated to be 0.44 million individuals [5].

Evidence has existed in the literature for decades that individuals with ID have poor oral hygiene and higher rate of gingivitis and periodontal disease (PD). Oral hygiene has been implicated as a casual factor in the development of PD in ID individuals. These subjects have the highest unattended dental treatment needs as compared with general population [6-12]. The oral health of the ID subjects is directly dependent upon their physical and mental abilities, their cooperativeness, and motivation of the support staff. Maintenance of good oral hygiene is difficult for many ID individuals, as frequently they lack muscular coordination and recognition of the importance of brushing and flossing. In many instances, the oral hygiene care of these subjects becomes the responsibility of another person, generally parent, guardian, or caregiver. As we know, oral hygiene has strong biological, psychological, and social projections on individuals well-being. Individuals with ID deserve the same opportunities for oral health and hygiene as those who are healthy. Unfortunately oral health care is one of the greatest unattended health needs of individuals with ID. It has been observed that oral health disparities among ID subjects were associated with practical difficulties in acceptance of routine treatment sessions, inadequate skills of dentists and hygienists to provide dental care, lack of effective preventive strategies to minimize the need for this care and negative attitudes, and lack of supervision by the parent, guardian, or caregiver toward oral health. These problems may be more profound in rural areas where availability, accessibility, and affordability of health services may be issue of disquiet $[7,13$, 14].

In India, despite the Persons with Disabilities Act (1995) and the National Trust Act (1999) enacted for persons with ID to enable and empower them, there is no legislation to date that makes a provision of dental services to the disabled population. Scientific research on ID in India has been predominant in the form of case reports. There is still a paucity of the literature on ID subjects [15]. There are a few reports on the oral hygiene status and periodontal status of the ID population from India, but none of those studies has explored the periodontal treatment needs of this population. The increase in degree of ID negatively influences oral hygiene status and periodontal status, which subsequently leads to increase in periodontal treatment needs. Lack of this important data is a serious limitation for provision of adequate treatment needs to this underprivileged population.
This study therefore intended to determine the oral hygiene status, periodontal status, and periodontal treatment needs and investigated the influence of various sociodemographic and clinical variables (age, gender, location, institution type, parent's education, SES, and degree of ID level) among multicentric institutionalized ID subjects in Kolhapur district, Maharashtra, India.

\section{Materials and Methods}

A cross-sectional descriptive survey was conducted in 610 ID subjects, aged 5-55 years, attending seven different ID institutes in Kolhapur district, Maharashtra, India. Survey was scheduled from November 2013 to February 2014. Subjects who were present at institutes on the days of the survey were included and those who were aggressive, noncooperative, and severely disabled patients were excluded from the study. Of the total 610 ID subjects seven were absent, 18 were noncooperative, and nine were systemically ill (severe detrimental disorders like cardiac defects). Hence, they were excluded from the study; thus the final sample size obtained was 576 . The ethical approval for conduct of the study was availed from Ethical Committee for Research of Tatyasaheb Kore Dental College \& Hospital, Kolhapur. A specially designed questionnaire proforma was prepared according to the need of study. The first part of the proforma consisted of collection of details regarding demographic information, location, SES, degree of ID, oral hygiene practices, and previous visit to the dentists. Information was collected by means of personal interviews with subject and parents or caregivers administered by examiner.

The IQ of the subjects was registered from the subject's records in the institute. The SES was recorded according to Kuppuswamy's SES scale. This scale takes into account education, occupation, and income of the family to categorize families into upper, upper middle, lower middle, upper lower, and lower SES [16]. The second part of the proforma consisted of the assessment of oral hygiene status, periodontal status, and periodontal treatment needs. A brief description of both the indices is as follows.

2.1. Oral Hygiene Status. Oral hygiene status was assessed by Simplified Oral Hygiene Index (OHI-S) which has two components, the Debris Index-Simplified (DI-S) and the Calculus Index-Simplified (CI-S), which are calculated separately and are summed up to get OHI-S for an individual. The examination was done using mouth mirror and explorer. The interpretation of index is as follows: good -0 to 1.2 , fair -1.3 to 3.0 , and poor-3.1 to 6.0 [17].

2.2. Periodontal Status and Periodontal Treatment Needs. The Community Periodontal Index of Treatment Needs (CPITN) was used to record periodontal status and periodontal treatment needs; it was assessed by CPITN probe [18]. The periodontal status was expressed in terms of Community Periodontal Index (CPI) code and loss of attachment score (LOA). Subjects below the age of 15 years were assessed for bleeding and calculus only as recording of periodontal 
pockets would be overestimated in this population because of false pockets. Hence, these subjects were not assessed for presence of periodontal pocket and LOA. The specified index teeth for CPITN index were 16, 11, 26, 36, 31, and 46. Pocket depths were measured at six sites around each tooth (mesial, middle, and distal on vestibular and lingual/palatal surfaces). The highest CPI and LOA scores for the sextants examined in a person were taken as the respective CPI and LOA for the person. The subjects were classified into different treatment need categories according to the highest scores which were recorded during the examination, as was recommended. The assessment was made as follows: code 0: healthy/TN0 (no need for treatment); code 1: bleeding on probing/TN1 (need for instruction to improve oral hygiene); code 2: supraor subgingival calculus found/TN2 (need for instruction in oral hygiene and calculus removal); code 3: pocket 4$5 \mathrm{~mm}$ deep/TN2 (need for instruction in oral hygiene and calculus removal and/or scaling and root planning); and code 4: pocket $6 \mathrm{~mm}$ deep or more/TN3 (need for instruction in oral hygiene, calculus removal, and complex periodontal treatment).

Throughout the study only one examiner conducted the examination and the assistant, who was trained, recorded the data. Dental team comprised of one examiner, two assistants, and a local health worker. 10 percent of the subjects examined each day were selected randomly to be reexamined and results were verified with initial exam to assess intraexaminer reliability [19]. Intraexaminer reliability for various indices was assessed using kappa statistic which was 92.3\%. Examination was carried out in school classrooms and premises. In all locations, natural light was used and the subjects were placed in such way that maximum illumination was obtained.

2.2.1. Statistical Analysis. Data was analyzed using the Statistical Package for Social Sciences (16.0 J for Windows; SPSS Japan). The mean and standard deviation were used to describe the pattern of oral hygiene status, periodontal status, and periodontal treatment needs among the different ID groups on bases of age, etiology of disability, and degree of ID. One-way analysis of variance (ANOVA) was used to test differences in mean scores of oral hygiene status. Multiple linear stepwise regression analysis was executed to analyze the associations of various sociodemographic and clinical variables with the oral hygiene indicators. Multiple logistic regression analysis was executed to test the associations of various independent variables with the oral hygiene status, periodontal status, and periodontal treatment needs scores. The continuous data were dichotomized for cross-tabulation and logistic regression analysis. The odds ratios (OR) with 95\% confidence intervals (95\% CI) were used to estimate the relative risk of the various variables for the occurrence of poor oral hygiene, periodontal disease, and periodontal treatment needs. Only those subjects aged 15 or more years were included in the multivariate analysis for occurrence of periodontal disease. For the purpose of multiple logistic regression analysis, response variables, namely, OHI-S, periodontal status, and periodontal treatment needs, were transformed to binary variables as oral hygiene status [good (good + fair) versus poor], periodontal status in CPI codes [fair (code $0+$ code $1+$ code 2 ) versus poor (code $3+$ code 4)], and periodontal treatment needs [low (TN0 + TN1 + TN2) versus high (TN3)]. Further independent variables were dichotomized as follows: age [young $<30$ years versus old $\geq 30$ years], gender [female versus male], education of mother and father [low (primary + secondary) versus high (undergraduate + postgraduate)], degree of ID [mild (mild + moderate) versus severe (severe + profound)], SES [low (lower middle + upper lower + lower class) versus high (upper + upper middle)], location [residential (subjects residing in the institute hostel) versus nonresidential (subjects residing with parents from urban and rural location)], and institution type [aided versus unaided]. The level of significance was set at $P<0.05$.

\section{Results and Discussion}

3.1. Results. This descriptive cross-sectional survey was conducted to determine the oral hygiene status, periodontal status, and periodontal treatment needs among the ID subjects attending the various ID institutes in Kolhapur district, Maharashtra, India.

Table 1 illustrates the general profile of the study population. It shows distribution of study subjects according to age, gender, location, degree of ID, education of parents, SES, and developmental disability type. There were 576 study subjects having age range from 5 to 55 with mean age of $17.50 \pm 7.4$ years. The maximum number of study subjects (47.2\%) belongs to $16-25$ years of age. There were 401 (69.6\%) males and 175 (30.4\%) females. SES was assessed by Kuppuswamy's classification; according to this classification $423(73.4 \%)$ subjects were belonging to upper lower and lower SES. Among the total 576 subjects, subjects with mild, moderate, severe, and profound levels of ID were 138 (24\%), 228 (39.60\%), 159 (27.60\%), and 51 (8.90\%), respectively. A residential subject (who resides at hostel) was 149 (25.90\%), while a nonresidential subject (who resides with parents from rural and urban location) was 427 (74.2\%). It was observed that $287(49.80 \%)$ of subjects' mothers were illiterate or had primary level of education.

Figure 1 signifies oral hygiene practices among ID subjects. It was observed that $416(72.2 \%)$ of subjects parents were not aware of oral hygiene status of subject. 550 (95.5\%) of subjects were visiting irregularly (those who had never visited and visit only when there is problem) the dentist, while only $26(4.5 \%)$ of subjects visit regularly every six months the dentist. 371 (64.5\%) subjects use brush to clean their teeth, among these subjects $182(43.9 \%)$ of subjects were dependent on their parents and caregivers for assistance. 196 (34\%) of subjects use finger as aid to clean their teeth.

Table 2 shows distribution oral hygiene status, periodontal status, and periodontal treatment needs according to different degrees of ID.

3.1.1. Oral Hygiene Status. It was observed that of total subjects with good, fair, and poor oral hygiene status were 5.73\%, 
TABLE 1: General profile of the ID subjects (all figures in percentage).

\begin{tabular}{|c|c|c|c|c|c|}
\hline \multirow{2}{*}{ Parameters } & \multicolumn{5}{|c|}{ Degree of ID } \\
\hline & Mild (\%) & Moderate (\%) & Severe (\%) & Profound (\%) & Total (\%) \\
\hline \multicolumn{6}{|l|}{ Age (years) } \\
\hline $5-15$ & 14.1 & 14.1 & 9.2 & 3.1 & 40.5 \\
\hline $16-25$ & 8.3 & 18.9 & 15.1 & 4.9 & 47.2 \\
\hline $26-35$ & 1.6 & 5.2 & 2.8 & 0.5 & 10.1 \\
\hline 36 and above & 0.2 & 1.4 & 0.5 & 0.2 & 2.3 \\
\hline \multicolumn{6}{|l|}{ Gender } \\
\hline Male & 16.1 & 28.5 & 19.3 & 5.7 & 69.6 \\
\hline Female & 8 & 11.1 & 8.3 & 3 & 30.4 \\
\hline \multicolumn{6}{|l|}{ Location } \\
\hline Residential & 5.4 & 9.5 & 8.5 & 2.4 & 25.9 \\
\hline Nonresidential & 18.8 & 30 & 19.1 & 6.2 & 74.2 \\
\hline \multicolumn{6}{|l|}{ Education of father } \\
\hline Primary & 10.2 & 20.1 & 15.1 & 4.3 & 49.8 \\
\hline Secondary & 7.8 & 14.6 & 8 & 3.6 & 34 \\
\hline $\mathrm{UG}$ & 5.7 & 4.9 & 4.5 & 0.7 & 15.8 \\
\hline PG & 0.3 & 0 & 0 & 0 & 0.3 \\
\hline \multicolumn{6}{|l|}{ Education of mother } \\
\hline Primary & 4.9 & 10.4 & 8 & 1.9 & 25.2 \\
\hline Secondary & 8.9 & 18.6 & 12.5 & 4.7 & 44.6 \\
\hline UG & 8.3 & 8.3 & 5.9 & 1.7 & 24.3 \\
\hline PG & 2.1 & 2.3 & 1.2 & 0.3 & 5.9 \\
\hline \multicolumn{6}{|l|}{ SES } \\
\hline Upper class & 0.9 & 0.2 & 0.2 & 0 & 1.2 \\
\hline Upper middle class & 1.4 & 1.7 & 1.6 & 0.3 & 5 \\
\hline Lower middle class & 7.5 & 6.4 & 5 & 1.4 & 20.3 \\
\hline Upper lower & 12 & 25 & 16 & 5.4 & 58.3 \\
\hline Lower & 2.4 & 6.3 & 4.9 & 1.6 & 15.1 \\
\hline \multicolumn{6}{|l|}{ Developmental disability type } \\
\hline Cerebral palsy & 19.7 & 31.1 & 32.8 & 16.4 & 31.8 \\
\hline Down's syndrome & 29.9 & 35.1 & 23.4 & 11.7 & 40.1 \\
\hline Autism & 11.5 & 46.2 & 26.9 & 15.4 & 13.5 \\
\hline Others (seizure disorders, congenital defects, social deprivation, etc.) & 7.1 & 42.9 & 32.1 & 17.9 & 14.6 \\
\hline
\end{tabular}

$43.23 \%$, and $51.04 \%$, respectively. Subjects of mild, moderate, severe, and profound degrees of ID groups had poor oral hygiene status score of $35.51 \%, 54.82 \%, 57.86 \%$, and $54.90 \%$, respectively.

\subsubsection{Periodontal Status}

CPI Score. The proportion of subjects with a healthy periodontium was only $0.17 \%$, while the subjects with presence of bleeding on probing (BOP) were $7.64 \%$. Presence of calculus was shown by $49.31 \%$ of subjects. The proportion of subjects with periodontal (PDL) pocket of 4-5 $\mathrm{mm}$ and PDL pocket of $6 \mathrm{~mm}$ was $22.78 \%$ and $15.10 \%$, respectively.

LOA Score. The proportion of subjects with LOA score of 0-3 mm, 4-5 mm, 6-8 mm, and 9-11 $\mathrm{mm}$ were $0.17 \%, 7.64 \%$, $73.78 \%$, and $18.4 \%$, respectively.
3.1.3. Periodontal Treatment Needs. The proportion of all subjects who were categorized as in need of oral prophylaxis (TN2) was $76.47 \%$, while that in need for complex periodontal treatment was $15.63 \%$.

Table 3 shows mean OHI-S, periodontal status, and periodontal treatment needs scores among the ID groups. There was statistically significant difference $(P<0.0001)$ among the groups for oral hygiene status, periodontal status, and periodontal treatment needs. Among the groups, subjects with profound level of ID had the highest mean OHI-S, periodontal status, and periodontal treatment needs score of $4.47 \pm 1.04,2.84 \pm 0.78$, and $2.25 \pm 0.44$, respectively. There was a definitive trend where mean score for all indices tended to increase gradually with increase in degree of ID.

Table 4 reveals that subject with another developmental disability (seizures, congenital defects, social deprivation, etc.) had higher mean OHI-S score compared with other 
TABLE 2: Revealing distribution oral hygiene status, periodontal status, and periodontal treatment needs according to degree of ID.

\begin{tabular}{|c|c|c|c|c|c|}
\hline \multirow{2}{*}{ Parameters } & \multicolumn{5}{|c|}{ Degree of ID } \\
\hline & Mild (\%) & Moderate (\%) & Severe (\%) & Profound (\%) & Total (\%) \\
\hline \multicolumn{6}{|c|}{ Oral hygiene status } \\
\hline Good & 10.14 & 6.58 & 1.89 & 1.96 & 5.73 \\
\hline Fair & 54.35 & 38.6 & 40.25 & 43.14 & 43.23 \\
\hline Poor & 35.51 & 54.82 & 57.86 & 54.90 & 51.04 \\
\hline \multicolumn{6}{|c|}{ Periodontal status } \\
\hline \multicolumn{6}{|l|}{ CPI score } \\
\hline Code 0 & 0.72 & 0 & 0 & 0 & 0.17 \\
\hline Code 1 & 12.32 & 9.21 & 3.77 & 0 & 7.64 \\
\hline Code 2 & 64.49 & 45.61 & 44.65 & 39.22 & 49.31 \\
\hline Code 3 & 19.57 & 28.95 & 30.19 & 37.25 & 27.78 \\
\hline Code 4 & 2.90 & 16.23 & 21.38 & 23.53 & 15.10 \\
\hline \multicolumn{6}{|c|}{ Loss of attachment score } \\
\hline $0-3 \mathrm{~mm}$ & 35.09 & 32.47 & 26.73 & 32.14 & 0.17 \\
\hline $4-5 \mathrm{~mm}$ & 50.88 & 45.45 & 43.56 & 42.86 & 7.64 \\
\hline $6-8 \mathrm{~mm}$ & 12.28 & 14.29 & 27.72 & 21.43 & 73.78 \\
\hline $9-11 \mathrm{~mm}$ & 1.75 & 7.79 & 1.98 & 3.57 & 18.4 \\
\hline \multicolumn{6}{|c|}{ Periodontal treatment needs } \\
\hline TN 0 & 0.72 & 0 & 0 & 0 & 0.17 \\
\hline TN 1 & 11.59 & 9.21 & 3.77 & 0 & 7.47 \\
\hline TN 2 & 83.33 & 74.56 & 74.84 & 76.47 & 76.47 \\
\hline TN 3 & 4.35 & 16.23 & 21.38 & 14 & 15.63 \\
\hline
\end{tabular}

All figures are in percentage.

TABLE 3: Revealing mean OHI-S, periodontal status, and periodontal treatment needs scores among the different degrees of ID.

\begin{tabular}{|c|c|c|c|c|}
\hline Degree of ID & $N$ & $\begin{array}{c}\text { OHI-S } \\
\text { Mean (SD) }\end{array}$ & $\begin{array}{c}\text { Periodontal status (CPI) } \\
\text { Mean (SD) }\end{array}$ & $\begin{array}{c}\text { Periodontal treatment needs } \\
\text { Mean }(\mathrm{SD})\end{array}$ \\
\hline Mild & 138 & $2.34(1.04)$ & $2.12(0.67)$ & $1.91(0.42)$ \\
\hline Moderate & 228 & $3.00(1.11)$ & $2.52(0.87)$ & $2.07(0.50)$ \\
\hline Severe & 159 & $3.77(0.9)$ & $2.69(0.84)$ & $2.18(0.47)$ \\
\hline Profound & 51 & $4.47(1.04)$ & $2.84(0.78)$ & $2.25(0.44)$ \\
\hline \multicolumn{2}{|c|}{ ANOVA } & $\begin{array}{l}F=75.932 \\
\mathbf{P}<\mathbf{0 . 0 0 0 1}\end{array}$ & $\begin{array}{l}F=16.231 \\
\mathbf{P}<\mathbf{0 . 0 0 0 1}\end{array}$ & $\begin{array}{l}F=10.385 \\
\mathbf{P}<\mathbf{0 . 0 0 0 1}\end{array}$ \\
\hline
\end{tabular}

TABLE 4: Mean OHI-S scores among the different developmental disability groups.

\begin{tabular}{lcc}
\hline $\begin{array}{l}\text { Developmental } \\
\text { disability } \\
\text { type }\end{array}$ & $N$ & $\begin{array}{c}\text { OHI-S } \\
\text { Mean (SD) }\end{array}$ \\
\hline Cerebral palsy & 61 & $3.2(1.15)$ \\
Down's syndrome & 77 & $3.06(1.33)$ \\
Autism & 26 & $2.88(1.21)$ \\
Others & 28 & $3.9(1.04)$ \\
\hline
\end{tabular}

ANOVA: $F=4.024 ; \mathbf{P}$ value $=\mathbf{0 . 0 0 8}$.

developmental disability types. The mean OHI-S score in descending order was $3.9 \pm 1.04$ (other developmental disabilities), CP (3.2 \pm 1.15$)$, DS (3.06 \pm 1.33$)$, and autistic group $(2.88 \pm 1.21)$ (with significance of $P=0.008)$.
Table 5 shows that multiple linear regression analysis was executed to estimate the linear relationship between the dependent variable OHI-S and various independent variables (age, gender, education of mother, education of father, SES, degree of ID, and location). It revealed that the best predictors in the descending order for OHI-S score as dependent variable were age, degree of ID, SES, and location.

Table 6 reveals distribution of categorized variables among ID groups for the purpose of multiple logistic regression analysis.

Table 7 reveals that the most important predictors for oral hygiene status, periodontal status, and periodontal treatment needs were older age, severe degree of ID, low SES, residential location of subjects, low education of mother, and unaided institutions. OR for poor oral hygiene status, periodontal status, and increased periodontal treatment needs was significantly higher among subjects with older age as compared 
TABLE 5: Multiple linear regression with OHI-S score as dependent variable.

\begin{tabular}{lcccc}
\hline Predictor & Coef. & SE coef. & $T$ & $P$ \\
\hline Constant & -0.6556 & 0.4175 & -1.57 & 14.84 \\
Age & 0.074482 & 0.00502 & -1.47 & 0.117 \\
Gender & -0.11547 & 0.07869 & 1.13 & 0.143 \\
Education of mother & 0.07883 & 0.07002 & 0.41 & 0.261 \\
Education of father & 0.02763 & 0.0676 & 3.6 & 0.683 \\
SES & 0.25226 & 0.07012 & 15.44 & $<\mathbf{0 . 0 0 0 1}$ \\
Degree of ID & 0.61745 & 0.03999 & 2.13 & $<\mathbf{0 . 0 0 0 1}$ \\
Location & 0.09537 & 0.04467 & $R$-Sq(adj) $=$ & 0.033 \\
\hline$S=0.8558$ & $R$-Sq $=$ & $51.4 \%$ & $50.8 \%$ \\
\hline
\end{tabular}

TABLE 6: Distribution of categorized data.

\begin{tabular}{llc}
\hline Categorized variable & Categories & $N(\%)$ \\
\hline \multirow{2}{*}{ Age } & Young: <30 years & $528(91.67 \%)$ \\
& Old: $\geq 30$ years & $(8.33 \%)$ \\
\hline \multirow{2}{*}{ SES } & High: [upper + upper middle] & $36(6.25 \%)$ \\
& Low: [upper lower + lower middle + lower class] & $540(93.75 \%)$ \\
Fathers' education & High: [undergraduate + postgraduate] & $173(30.03 \%)$ \\
\hline \multirow{2}{*}{ Mothers' education } & Low: [primary + secondary] & $403(69.97 \%)$ \\
\hline \multirow{2}{*}{ Degree of ID } & High: [undergraduate + postgraduate] & $93(16.14 \%)$ \\
& Low: [primary + secondary] & $483(83.86 \%)$ \\
Oral hygiene status (OHI-S) & Mild: [mild + moderate] & $366(63.54 \%)$ \\
\hline Periodontal status (CPI code) & Severe: [severe + profound] & $210(36.46 \%)$ \\
\hline \multirow{2}{*}{ Periodontal treatment needs (CPITN score) } & Good: [good + fair] & $282(48.96 \%)$ \\
\hline Institution type & Poor: [Poor] & $294(51.04 \%)$ \\
\hline
\end{tabular}

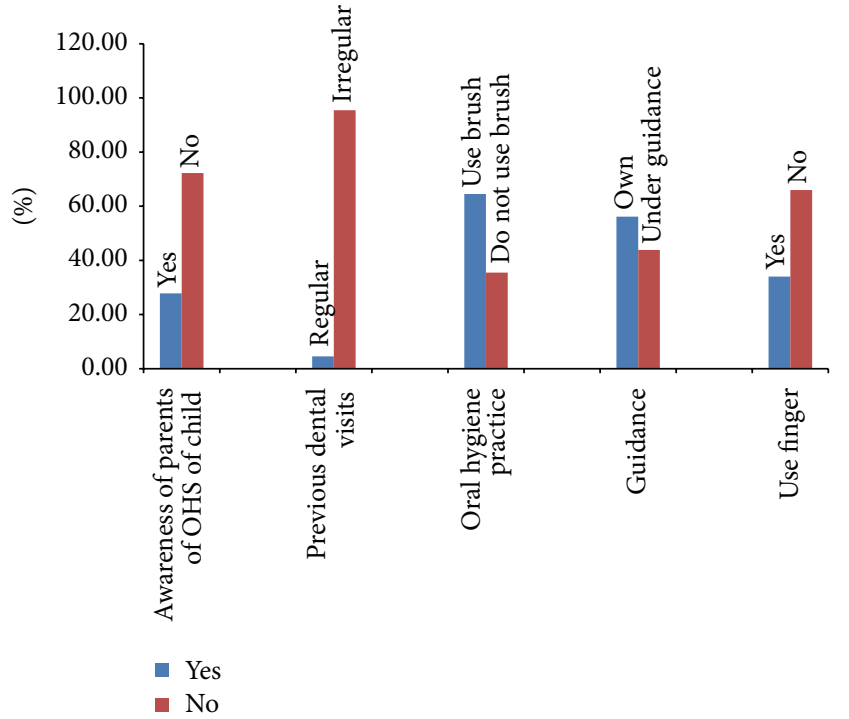

FIGURE 1: Oral hygiene practices. with younger age $(P<0.0001)$, among subjects with severe degree of ID as compared with mild degree of ID $(P<0.0001)$ and subjects with lower SES as compared to higher SES. A similar pattern was observed for these dependent variables, where OR was significantly higher among subjects with less educated mothers, among those subjects who were residential as compared with nonresidential ones and subjects who enrolled in unaided institutes as compared with government aided institutes.

3.2. Discussion. Individuals with ID constitute a unique but heterogeneous population, which includes a great variety of mental and developmental disorders as well as congenital syndromes. It has been observed consistently that oral health checks lead to detection of unmet treatment needs and lead to targeted actions to address dental treatment needs [7]. The unmet dental treatment for this group is due in part to the underlying cause of their disability and in part to predisposing and interrelated factors such as poverty, illiteracy, and other social determinants. Improved oral health is likely to 
TABLE 7: Logistic regression: odds ratio (OR) and 95\% confidence interval (CI) for oral hygiene status, periodontal status, and periodontal treatment needs according to age, gender, education of father, education of mother, degree of ID, SES, location, and institution type.

\begin{tabular}{|c|c|c|c|}
\hline Independent variable & $\begin{array}{l}\text { Oral hygiene status } \\
\text { OR }(95 \% \mathrm{CI})\end{array}$ & $\begin{array}{l}\text { Periodontal status } \\
(\mathrm{CPI}) \text { OR }(95 \% \mathrm{CI}) \\
\end{array}$ & $\begin{array}{c}\text { Periodontal treatment } \\
\text { needs OR (95\% CI) }\end{array}$ \\
\hline Age: young versus old & $\begin{array}{c}6.36(2.818-14.368) \\
(\mathbf{P}<\mathbf{0 . 0 0 0 1})\end{array}$ & $\begin{array}{c}40.609(9.581-172.12) \\
(\mathbf{P}<\mathbf{0 . 0 0 0 1})\end{array}$ & $\begin{array}{c}16.087(7.694-33.637) \\
(\mathbf{P}<\mathbf{0 . 0 0 0 1})\end{array}$ \\
\hline Gender: male versus female & $\begin{array}{c}0.779(0.522-1.161) \\
(P=0.219)\end{array}$ & $\begin{array}{c}0.855(0.573-1.275) \\
(P=0.442)\end{array}$ & $\begin{array}{c}1.031(0.598-1.778) \\
(P=0.914)\end{array}$ \\
\hline Father education: low versus high & $\begin{array}{c}0.634(0.385-1.045) \\
(P=0.074)\end{array}$ & $\begin{array}{c}0.685(0.414-1.134) \\
(P=0.141)\end{array}$ & $\begin{array}{c}0.715(0.336-1.522) \\
(P=0.384)\end{array}$ \\
\hline Mother education: low versus high & $\begin{array}{c}1.321(0.671-2.602) \\
(P=0.42)\end{array}$ & $\begin{array}{c}1.306(0.668-2.554) \\
(P=0.436)\end{array}$ & $\begin{array}{c}1.16(0.417-3.228) \\
(P=0.776)\end{array}$ \\
\hline ID: mild versus severe & $\begin{array}{c}4.39(2.958-6.514) \\
(\mathbf{P}<\mathbf{0 . 0 0 0 1})\end{array}$ & $\begin{array}{c}2.191(1.517-3.165) \\
(\mathbf{P}<\mathbf{0 . 0 0 0 1})\end{array}$ & $\begin{array}{c}2.621(1.568-4.38) \\
(\mathbf{P}<\mathbf{0 . 0 0 0 1})\end{array}$ \\
\hline SES: high versus low & $\begin{array}{c}3.75(1.46-9.61) \\
(\mathbf{P}<\mathbf{0 . 0 0 6}) \\
\end{array}$ & $\begin{array}{c}2.06(0.80-5.26) \\
(P=0.133)\end{array}$ & $\begin{array}{c}13.15(1.34-128.9) \\
(\mathbf{P}=\mathbf{0 . 0 2})\end{array}$ \\
\hline Location: nonresidential versus residential & $\begin{array}{c}1.381(0.829-2.301) \\
(P=0.216)\end{array}$ & $\begin{array}{c}1.052(0.642-1.725) \\
(P=0.84)\end{array}$ & $\begin{array}{c}1.087(0.559-2.115) \\
(P=0.805)\end{array}$ \\
\hline Institution type: aided versus unaided & $\begin{array}{c}1.244(0.777-1.993) \\
(P=0.363)\end{array}$ & $\begin{array}{c}0.778(0.492-1.23) \\
(P=0.283)\end{array}$ & $\begin{array}{c}1.021(0.543-1.921) \\
(P=0.948)\end{array}$ \\
\hline
\end{tabular}

$\mathrm{OR}=$ odds ratio; $\mathrm{CI}=$ confidence interval.

lead to improved quality of life for both the individuals with ID and their families. The majority of the studies conducted on ID population have provided data regarding the oral hygiene status and periodontal status of assorted disability groups or assessment with normal population. In the literature there are few studies about periodontal treatment needs of ID individuals $[10-12,20,21]$, while in India there is no study which has provided data about periodontal treatment needs of this vulnerable population. As a result of growing concern about the oral health of ID subjects in India, the aim of this cross-sectional study was to investigate the oral hygiene status, periodontal status, and periodontal treatment needs of this population. The finding depicted the influence of various sociodemographic characters and clinical variables (age, gender, location, parent's education, SES, institution type, associated developmental disability, and degree of ID) on oral hygiene status, periodontal status, and periodontal treatment needs of ID subjects. In this study meticulous periodontal examinations were difficult, due to limitations in cooperation, low ability of concentration, communication problem, and hyperactive behavior of the subjects. However, bleeding response, probing depth, and presence or absence of calculus were carefully recorded.

Anders and Davis reviewed 27 studies of individuals with ID and reported that such individuals tend to have poorer oral hygiene and higher prevalence and greater severity of PD. Two subgroups at especially high risk for oral health problems are individuals with DS and people unable to cooperate with routine dental care [7]. Comparison between studies is difficult because of the lack of common indices. In general, oral cleanliness is less adequate and deteriorates more with age in subjects with ID, DS, and CP [22-25]. The process of developing gingival and PD in ID does not differ from nondisabled individuals. The main factor related to gingival and PD in ID subjects is the physical inadequacy of the mechanical plaque removal from the teeth. Limited motor skills, lack of knowledge of oral hygiene, and effective brushing technique in ID have resulted in the progression of inflammatory diseases.

In the present study the majority of subjects had never visited dentist. This could be due to their low SES, parental education, underestimation of treatment needs, and fear of dental procedures, along with the cost of dental care, which might have influenced dental service utilization. De Jongh et al. [26] reported that a significantly higher proportion of noninstitutionalized ID subjects in their study did not receive any routine dental care in comparison with healthy controls (53.1\% and $23.8 \%$, resp.) because noncooperation and communication problems were important barriers leading to a relatively low degree of quality dental care.

3.2.1. Oral Hygiene Status. The prevalence rate was $5.73 \%$, $43.23 \%$, and $51.04 \%$ for good, fair, and poor oral hygiene status, respectively. This was worse than that of a previous study conducted on ID subjects attending special schools in India $[25,27]$. The overall mean OHI-S score of ID subjects was found to be $3.17 \pm 1.226$, which was found to be better as compared with that of study by Kaur et al. $(4.956 \pm 1.236)$ in the age range of $10-90$ years [27], $(3.21 \pm 1.04)$ in the age range 14-17 years in a study by Ameer et al. [22], and $(3.80 \pm 1.42)$ in the age range of $8-19$ years in a study by Kumar et al. (2009) [25]. In the present study, the mean OHI-S score in descending order was $4.47 \pm 1.04$ (profound), $3.77 \pm 0.9$ (severe), $3.00 \pm 1.11$ (moderate), and $2.34 \pm 1.04$ (mild) for each ID group, respectively (with significant difference $(P<$ $0.0001)$ ). It indicates that mean OHI-S score for mild and moderate degrees of ID is less as compared with severe and profound degrees of ID. It could be because subjects with mild and moderate degrees of ID are able to understand the need for practicing good oral hygiene and are able to 
comprehend instructions given by care givers. The higher score among severe and profound degrees of ID could be due to their inefficiency to brush their teeth themselves or dependence of subjects for oral hygiene maintenance on parents or caregivers. It has also been observed that among ID subjects those who brushed with assistance (parent or caretaker) had diminutive better levels of oral hygiene than those who self-brushed. The mean OHI-S was found to be increasing with increase in age, being the highest for age group of 46 to 55 years, that is, $5.82 \pm 0.27$. The general increase in OHI-S scores with increase in age could be due to cumulative effect of plaque and calculus. This is consistent with previous studies $[4,22,23,25]$. The results of multiple linear regression and multiple logistic regression depicted a poor oral hygiene status in ID subjects with older age $(\mathrm{OR}=6.363)$, severe degree of ID $(\mathrm{OR}=4.39)$, low SES $(\mathrm{OR}=3.75)$, residential subjects $(\mathrm{OR}=1.381)$, less educated mother $(\mathrm{OR}=1.321)$, and unaided institutes $(\mathrm{OR}=1.244)$. The subjects with low SES and low level of parental education may lack awareness about the importance of oral health and its proper care. It has been observed that the majority of subjects' parents had low level of education. Hence, they may also be unable to access or afford the appropriate oral hygiene aids and health care facilities. This finding could be explained by the results from a study which revealed a higher percentage of subjects with good oral hygiene in upper SES group [28], while in a study by Tesini it was reported that SES has no significant impact on oral hygiene status of ID subjects [9]. In residential subjects, lack of knowledge and negligence among caregivers towards providing oral health care to ID subjects was major hurdle. Hence, although parents were having good educational background and residential location was in an urban area of the state, still subjects were having poor oral hygiene status. This is consistent with previous studies $[7,26$, 28].

Mean OHI-S score for different developmental disability types was higher for other developmental disabilities (i.e., seizures, congenital defects, social deprivation, etc.). This could be due to their underlying systemic condition and consumption of various medications for their systemic disorders such as Phenytoin sodium (seizure disorder), Nifedipine (Hypertension) and so forth. The use of multiple medications may produce a variety of side effects and drug interactions; which subsequently affects oral status. In CP subjects presence of malocclusion and disability in limbs in addition to ID is measure hurdle in achieving good oral hygiene [24].

3.2.2. Periodontal Status and Periodontal Treatment Needs. The ID subjects in the present study had overall poor periodontal status with high periodontal treatment needs. It has been observed that only $0.17 \%$ had healthy periodontal status, while bleeding on probing, calculus, shallow pockets, and deep pockets were found in $7.64 \%, 49.31 \%, 27.78 \%$, and $15.10 \%$, respectively. Among each ID group PDL pocket with $4-5 \mathrm{~mm}$ was observed to be higher in profound ID group $(37.25 \%)$, while in severe, moderate, and mild groups it was $30.19 \%, 28.95 \%$, and $19.57 \%$, respectively. In a similar study by Lucchese and Checchi the periodontal status in the study population was very poor. $66 \%$ of the subjects presented with a probing depth more than $3.5 \mathrm{~mm}, 29 \%$ showed the presence of bleeding or calculus, and only $5 \%$ showed a normal periodontal status [29].

Among ID groups LOA score of $6-8 \mathrm{~mm}$ was higher among severe $(27.72 \%)$ and profound $(21.43 \%)$ groups. Ameer et al. [22] observed the highest LOA score of $4-5 \mathrm{~mm}$ in ID individuals as compared with other disabled study populations. The higher incidence of $\mathrm{PD}$ can be attributed to the lack of manual dexterity among these disabled subjects. On the contrary Shaw et al. assessed manual dexterity in their study and showed that although periodontal health was poor among the group, it was not correlated with manual dexterity [30]. High prevalence of PD was detected in subjects with DS as compared with other disability groups. Several researchers have reported a significant correlation between DS and poor periodontal status. The greater severity of PD has been attributed in DS subjects to factors such as lower resistance to bacterial infection, malocclusion, traumatic occlusions, tooth morphology, and lack of normal mastication $[25,31,32]$.

The proportion of subjects requiring periodontal treatment in this study was very high. The most prevalent treatment need was TN2 (76.47\%). The complex periodontal care was required for $15.63 \%$ of subjects. Comparison with other studies indicated that periodontal treatment needs mainly fall into TN1 category. Denloye [11] observed that none of the children had healthy periodontium and all the children will need oral hygiene care with a significant percentage (8\%) requiring complex periodontal care.

Among ID group mean CPI score was the highest in profound degree $(2.84 \pm 0.78)$ as compared with severe $(2.69 \pm$ $0.84)$, moderate $(2.52 \pm 0.87)$, and mild $(2.12 \pm 0.67)$ degrees of ID $(P<0.0001)$. Similar observation was reported for mean CPITN score in descending order, that is, $2.25 \pm 0.44$ (profound), $2.18 \pm 0.47$ (severe), $2.07 \pm 0.50$ (moderate), and $1.91 \pm$ 0.42 (mild) among ID groups $(P<0.0001)$. Nematollahi et al. [20] observed that mean CPITN increased with age $(P=$ $0.01)$ and with the level of ID $(P=0.001)$. The treatments needed for most of the children were TN1 (74.42\%), followed by TN2 (23.64\%) and TN3 (1.16\%). Only, $0.78 \%$ of the population demonstrated healthy periodontal tissue. Mean CPITN was significantly higher in governmental centers than private ones $(P=0.02)$. Bhavsar and Damle [21] in their study observed that the bleeding and calculus components were higher than the healthy components in all the groups and almost all the children required treatment in the form of deep scaling and/or prophylaxis and oral hygiene instructions. Multivariate analysis elicited poor periodontal status with older age $(\mathrm{OR}=40.6,95 \% \mathrm{CI}=9.581-172.12)$, severe degree of ID $(\mathrm{OR}=2.621)$, low SES $(\mathrm{OR}=2.06,95 \% \mathrm{CI}=1.517-$ $5.26)$, and less educated mothers $(\mathrm{OR}=1.3)$ and in those who were residential $(\mathrm{OR}=1.052)$. Similarly increased periodontal treatment needs were observed with older age (OR $=16.08,95 \% \mathrm{CI}=7.694-33.637)$, severe degree of ID ( $\mathrm{OR}=$ $2.62)$, less educated mothers $(\mathrm{OR}=1.16)$, and low SES $(\mathrm{OR}=$ $13.15,95 \% \mathrm{CI}=1.34-128.9)$ and in residential location $(\mathrm{OR}=$ $1.087)$, females $(\mathrm{OR}=1.031)$, and unaided institutes $(\mathrm{OR}=$ $1.021)$. 
It indicates that in the present study the periodontal status and subsequently periodontal treatment needs were highly influenced by increasing age, severity of ID, lower SES, less education of mother, residential location associated disability, and unaided institutes. The relationship of increasing age and worsening periodontal status was reflected in CPI scores for periodontal pockets. This is consistent with other studies [22, 25]. In accordance with other studies, our study also showed that increase in the severity of ID resulted in worsening of periodontal condition and tooth loss. This is likely due to the continuous neglect towards personal and oral hygiene. Patients who were ID of severe and profound degrees had increased periodontal status scores. This was expected as while the degree of helplessness worsens, the ability of subjects to perform their daily activities reduces. However, Górski and Buczkowska-Radlińska in their study mentioned that the knowledge of dental prophylaxis and dental treatment aspects of the parents of moderately ID children did not reveal any significant impact on oral hygiene status and periodontal status [33]. Moreover a high correlation between poor oral hygiene and the development and progression of PD has been well documented and the role of poor oral hygiene as a risk factor of PD is well established [34]. A similar study by Svatun and Gjermo [10] observed the average oral hygiene and poor periodontal health except for patients in a few institutions where the nurses were trained to clean teeth regularly. Increased age, high degree of ID, seizure disorder, and DS were all elements that apparently contributed to impairment of periodontal status and to increased periodontal treatment needs. The preventive programs used in some institutions seemed to be effective as compared with noninstitutionalized subjects. However Tesini [9] observed that institutional status was a major determinant in oral hygiene conditions of the study group, as institutionalized ID individuals had significantly higher OHI-S scores than noninstitutionalized ID individuals. SES was not found to be a significant factor in the oral hygiene status of ID individuals. In present study SES was highly significant factor in the oral hygiene status, periodontal status, and periodontal treatment needs of subjects. A study by Kumar et al. observed that the oral hygiene status and periodontal status of ID population were influenced by disability type, degree of ID, disabled sibling, parent's education, and SES [25].

This study has highlighted important aspects in the oral hygiene status, periodontal status, and periodontal treatment needs of the ID institutionalized population. It has shown that poor oral health is a major problem for ID and the oral hygiene status of these subjects seemed to indicate a cumulative neglect of oral health. This study confirmed the need for strengthening organized preventive strategies and care for this population in India. The highly alarming situation needs immediate attention as oral hygiene status of these subjects is related to their social acceptability. Even though efforts have been made in the Western world to improve the oral health of these less fortunate subjects, less attention has been directed by the health authorities in India. The average oral hygiene status and periodontal status were poor, many of them requiring complex treatment except for subjects in a few institutions where the caregivers were trained to clean teeth regularly. Increased age, higher degree of ID, poor SES, residential location, and unaided institutes were all elements that apparently contributed to impairment of periodontal status and to increased treatment requirements. However, prevention should be the main objective because subjects with advanced ID are often anxious and uncooperative in the dental clinic. Hence, no dental treatment was provided except referral to the dental surgeon for emergency treatment, thereby precluding complex treatment. Hence more coordinative efforts between medical, dental, and social care sectors must be established to serve the needs of this underprivileged population.

This study was limited to the institutionalized ID subjects from Kolhapur district, Maharashtra, India, who participated in the screening on the day of oral health examination. It has been observed that the multifactorial influence was present on overall oral health of ID subjects. This study was dominated by male population. Age, ID, and SES were found to be highly significant. Results of this particular study should not be generalized to any other part of the country. Some subjects were not in institutes on the days of screening, some were very ill, and some were uncooperative, so they were excluded from study. Comparison between studies was difficult because of the lack of common indices. Although subjects had shown presence of pathological pockets below the age of 15, they were not assessed for poor periodontal conditions. Due to lack of cooperativeness and time constraint, other risk factors associated with ID, which subsequently lead to poor oral health, were not estimated like hereditary factors, consumption of medications, food intake habits, quality of water, and so forth. Despite all these factors the present study was able to achieve its goals in diagnosing prevalence of PD and periodontal treatment needs of this vulnerable population. More studies are essential in the future to eliminate disparities and provision for equal rights. Caregivers requested training in oral care and the use of dental services and support in dealing with ID subjects who are different than the normal population.

\section{Conclusion}

Subjects belonging to the ID groups indoctrinate behaviors under the influence of aptitude, surroundings, and interest of teachers, parents, and caretakers. The lack of attention leads to deprivation resulting in poor oral hygiene, subsequent $\mathrm{PD}$, and increased periodontal treatment needs. In order to achieve a satisfactory oral health a holistic and multidisciplinary approach is needed for these subjects. Management of ID should be included in dental curriculum and for this necessary training should be given. Constant motivation of the parent and caregivers is essential to bridge the gap.

\section{Conflict of Interests}

No potential conflict of interests relevant to this paper was reported. 


\section{Acknowledgment}

The authors sincerely thank all ID institutes of Kolhapur district, Maharashtra, India, for their constant help and support during the study.

\section{References}

[1] P. Gabre, T. Martinsson, and L. Gahnberg, "Incidence of, and reasons for, tooth mortality among mentally retarded adults during a 10-year period," Acta Odontologica Scandinavica, vol. 57, no. 1, pp. 56-61, 1999.

[2] American Psychiatric Association, Diagnostic and Statistical Manual of Mental Disorders, APA Press, Washington, DC, USA, 5th edition, 2013.

[3] L. Salvador-Carulla, G. M. Reed, L. M. Vaez-Azizi et al., "Intellectual developmental disorders: towards a new name, definition and framework for "mental retardation/intellectual disability” in ICD-11," World Psychiatry, vol. 10, no. 3, pp. 175180, 2011.

[4] P. K. Maulik, M. N. Mascarenhas, C. D. Mathers, T. Dua, and S. Saxena, "Prevalence of intellectual disability: a meta-analysis of population-based studies," Research in Developmental Disabilities, vol. 32, no. 2, pp. 419-436, 2011.

[5] Government of India, "Disabled persons in India," Tech. Rep. 485 (58/26/1), National Sample Survey Organisation, New Delhi, India, 2003.

[6] D. A. Tesini, "An annotated review of the literature of dental caries and periodontal disease in mentally retarded individuals," Special Care in Dentistry, vol. 1, no. 2, pp. 75-87, 1981.

[7] P. L. Anders and E. L. Davis, "Oral health of patients with intellectual disabilities: a systematic review," Special Care in Dentistry, vol. 30, no. 3, pp. 110-117, 2010.

[8] K. Gerreth and M. Borysewicz-Lewicka, "Epidemiological evaluation of gingivitis in special-care schoolchildren," Medycyna Wieku Rozwojowego, vol. 13, no. 4, pp. 283-291, 2009.

[9] D. A. Tesini, "Age, degree of mental retardation, institutionalization, and socioeconomic status as determinants in the oral hygiene status of mentally retarded individuals," Community Dentistry and Oral Epidemiology, vol. 8, no. 7, pp. 355-359, 1980.

[10] B. Svatun and P. Gjermo, "Oral hygiene, periodontal health and need for periodontal treatment among institutionalized mentally subnormal persons in Norway," Acta Odontologica Scandinavica, vol. 36, no. 2, pp. 89-95, 1978.

[11] O. O. Denloye, "Periodontal status and treatment needs of 12-15 year old institutionalized mentally handicapped school children in Ibadan, Nigeria," Odonto-Stomatologie Tropicale, vol. 22, no. 86, pp. 38-40, 1999.

[12] R. Kozak, "Dental and periodontal status and treatment needs of institutionalized mentally retarded children from the province of West Pomerania," Annales Academiae Medicae Stetinensis, vol. 50, no. 2, pp. 149-156, 2004.

[13] J. P. Morgan, P. M. Minihan, P. C. Stark et al., "The oral health status of 4,732 adults with intellectual and developmental disabilities," Journal of the American Dental Association, vol. 143, no. 8, pp. 838-846, 2012.

[14] S. Cumella, N. Ransford, J. Lyons, and H. Burnham, "Needs for oral care among people with intellectual disability not in contact with community dental services," Journal of Intellectual Disability Research, vol. 44, no. 1, pp. 45-52, 2000.
[15] B. Chavan and A. Rozatkar, "Intellectual disability in India: charity to right based," Indian Journal of Psychiatry, vol. 56, no. 2, pp. 113-116, 2014.

[16] K. Vijaya and E. Ravikiran, "Kuppuswamy's socio-economic status scale-updating income ranges for the year 2013," National Journal of Research in Community Medicine, vol. 2, no. 2, pp. 79-148, 2013.

[17] J. C. Greene and J. R. Vermillion, "The simplified oral hygiene index," Journal of the American Dental Association, vol. 68, pp. 7-14, 1964.

[18] J. Ainamo, D. Barmes, G. Beagrie, T. Cutress, J. Martin, and J. Sardo-Infirri, "Development of the World Health Organization (WHO) community periodontal index of treatment needs (CPITN)," International Dental Journal, vol. 32, no. 3, pp. 281291, 1982

[19] S. Eklund, I. J. Moller, and M. H. LeClercq, "Calibration of examiners for oral epidemiological surveys," Tech. Rep. ORH/EIS/EPID.93.1, World Health Organization, 1993.

[20] H. Nematollahi, A. Makarem, and A. R. Noghani, "Periodontal treatment needs amongst 9-14 year-old institutionalized mentally retarded children in Mashhad, Iran," Journal of Dentistry (Shiraz University of Medical Sciences), vol. 10, pp. 15-20, 2010.

[21] J. P. Bhavsar and S. G. Damle, "Dental caries and oral hygiene amongst 12-14 years old handicapped children of Bombay, India," Journal of the Indian Society of Pedodontics and Preventive Dentistry, vol. 13, no. 1, pp. 1-3, 1995.

[22] N. Ameer, R. Palaparthi, M. Neerudu, S. K. Palakuru, H. R. Singam, and S. Durvasula, "Oral hygiene and periodontal status of teenagers with special needs in the district of Nalgonda, India," Journal of Indian Society of Periodontology, vol. 16, no. 3, pp. 421-425, 2012.

[23] M. Jain, A. Mathur, L. Sawla et al., "Oral health status of mentally disabled subjects in India," Journal of Oral Science, vol. 51, no. 3, pp. 333-340, 2009.

[24] J. E. Pope and M. E. Curzon, "The dental status of cerebral palsied children,” Pediatric Dentistry, vol. 13, no. 3, pp. 156-162, 1991.

[25] S. Kumar, J. Sharma, P. Duraiswamy, and S. Kulkarni, "Determinants for oral hygiene and periodontal status among mentally disabled children and adolescents," Journal of Indian Society of Pedodontics and Preventive Dentistry, vol. 27, no. 3, pp. 151-157, 2009.

[26] A. De Jongh, C. van Houtem, M. van Der Schoof, G. Resida, and D. Broers, "Oral health status, treatment needs, and obstacles to dental care among noninstitutionalized children with severe mental disabilities in the Netherlands," Special Care in Dentistry, vol. 28, no. 3, pp. 111-115, 2008.

[27] S. Kaur, R. Malhotra, R. Malhotra, H. Kaur, V. S. Battu, and A. Kaur, "Oral hygiene status of mentally and physically challenged individuals living in a specialized institution in Mohali," Indian Journal of Oral Sciences, vol. 4, no. 1, pp. 17-22, 2013.

[28] F. A. Oredugba and Y. Akindayomi, "Oral health status and treatment needs of children and young adults attending a day centre for individuals with special health care needs," BMC Oral Health, vol. 8, no. 1, article 30, 2008.

[29] C. Lucchese and L. Checchi, "The oral status in mentally retarded institutionalized patients," Minerva Stomatologica, vol. 47, no. 10, pp. 499-502, 1998.

[30] L. Shaw, M. J. Shaw, and T. D. Foster, "Correlation of manual dexterity and comprehension with oral hygiene and periodontal status in mentally handicapped adults," Community Dentistry and Oral Epidemiology, vol. 17, no. 4, pp. 187-189, 1989. 
[31] T. W. Cutress, "Periodontal disease and oral hygiene in trisomy 21," Archives of Oral Biology, vol. 16, no. 11, pp. 1345-1355, 1971.

[32] S. S. Desai, "Down syndrome: a review of the literature," Oral Surgery, Oral Medicine, Oral Pathology, Oral Radiology, and Endodontics, vol. 84, no. 3, pp. 279-285, 1997.

[33] M. Górski and J. Buczkowska-Radlińska, “Teeth and periodontium status of moderately mentally retarded children and the health awareness of their parents," Annales Academiae Medicae Stetinensis, vol. 53, no. 2, pp. 92-99, 2007.

[34] P. E. Petersen and H. Ogawa, "Strengthening the prevention of periodontal disease: the WHO approach," Journal of Periodontology, vol. 76, no. 12, pp. 2187-2193, 2005. 


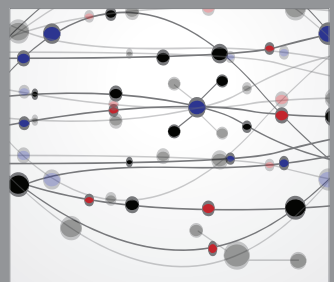

The Scientific World Journal
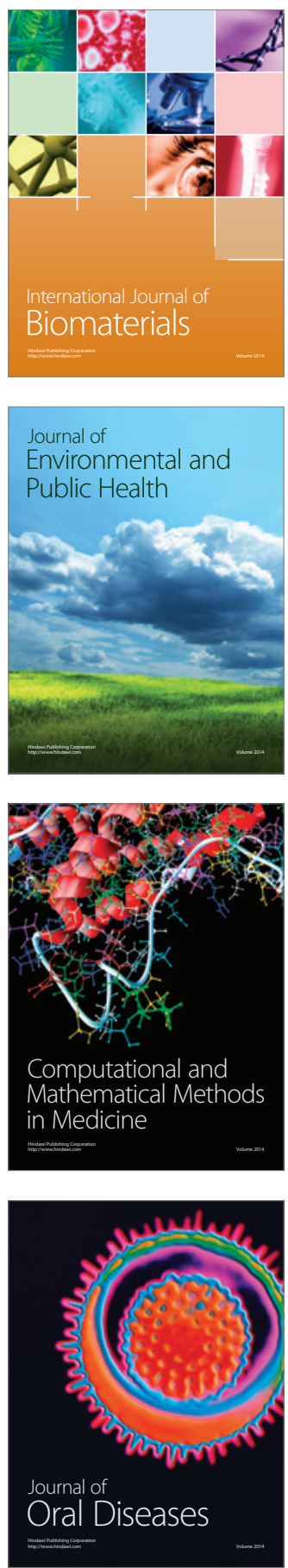
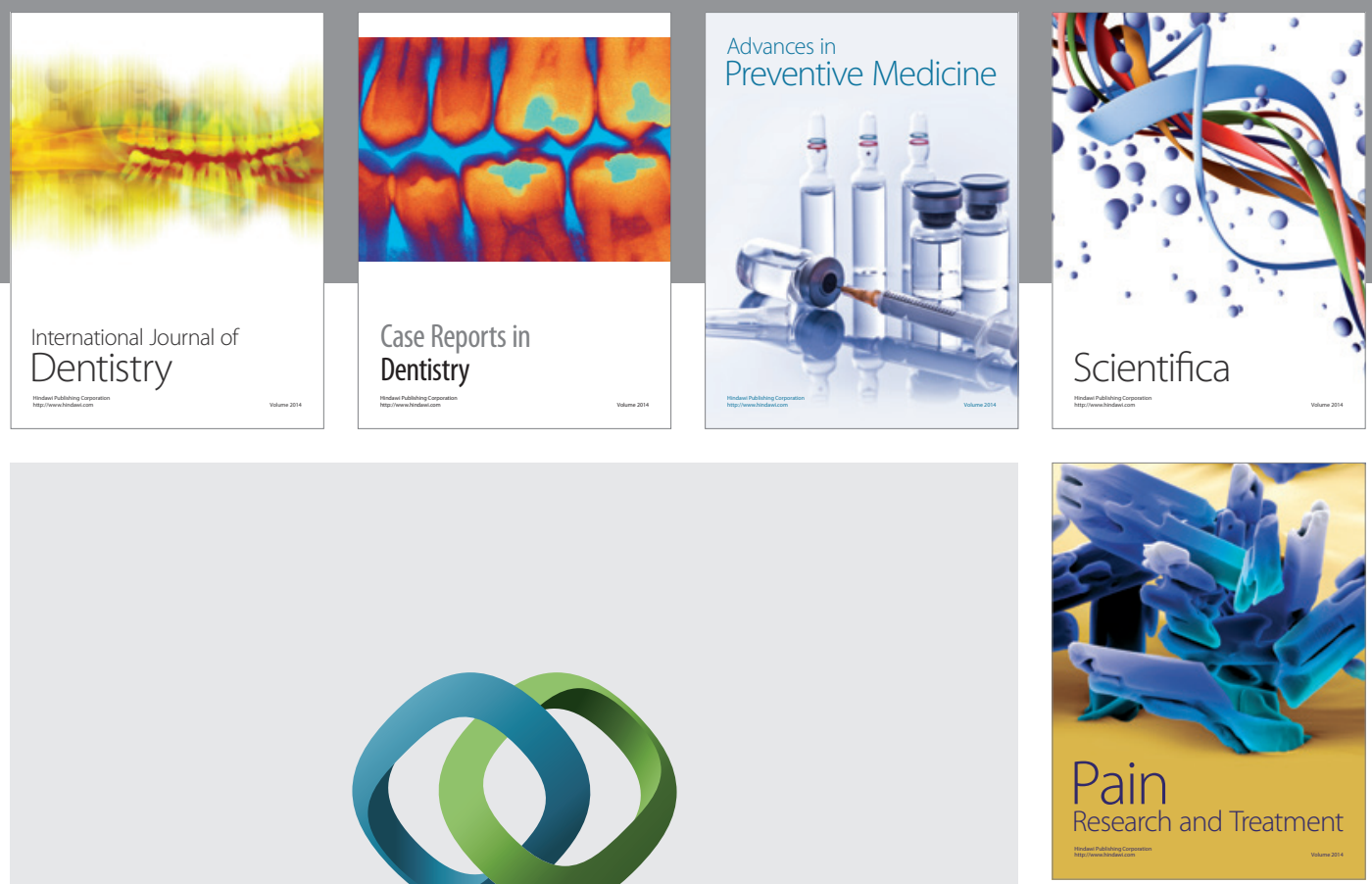

\section{Hindawi}

Submit your manuscripts at

http://www.hindawi.com
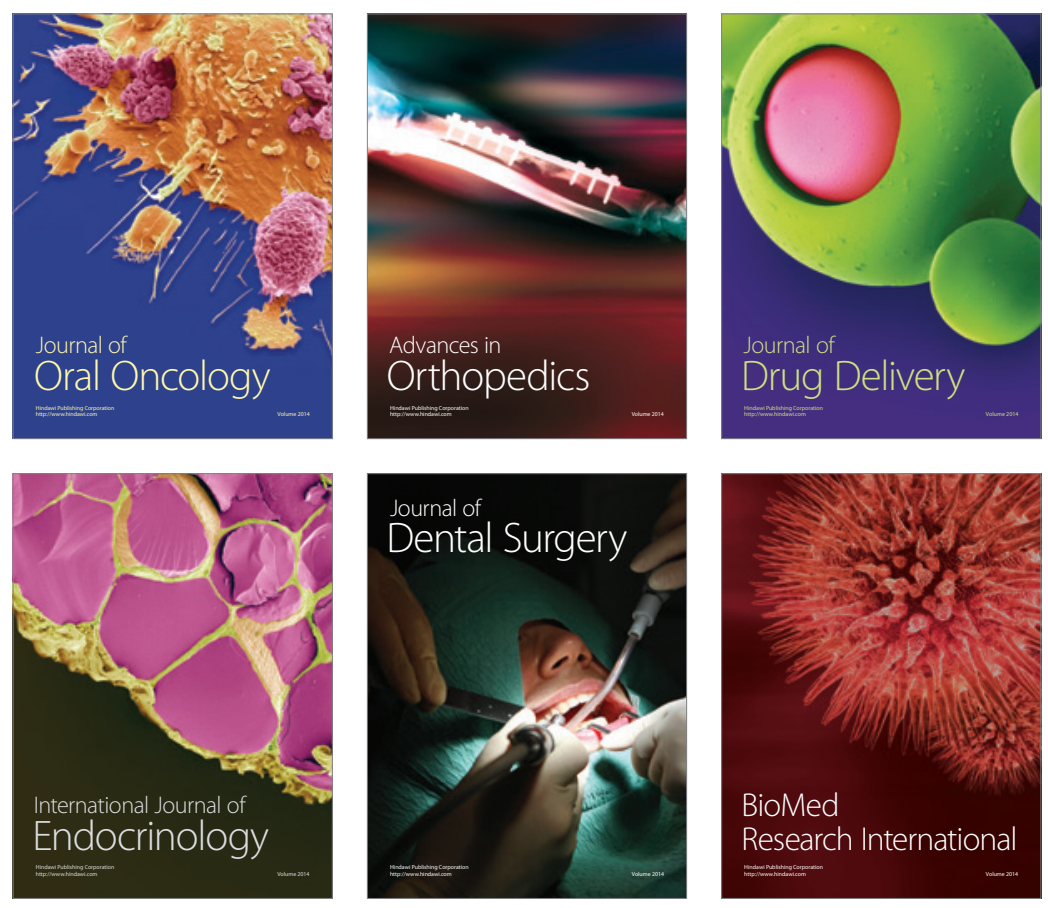

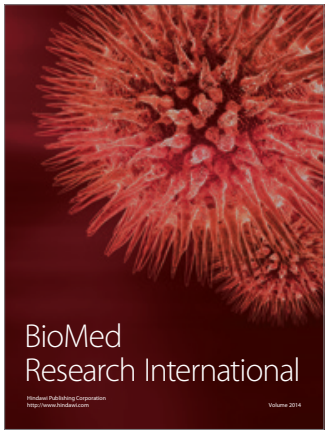

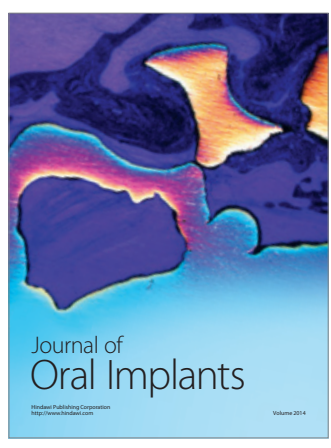
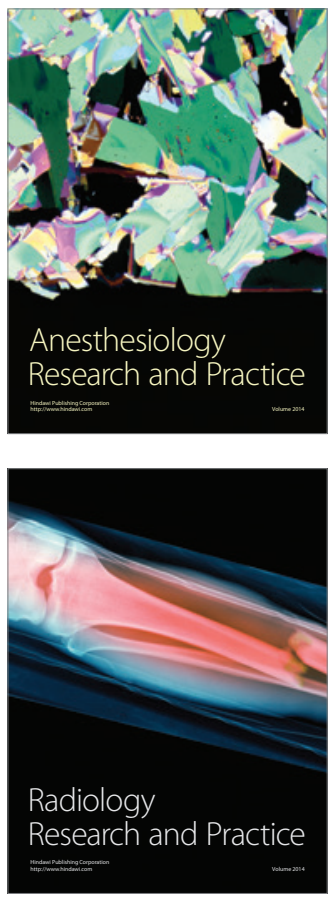